\title{
In-medium meson effects on the equation of state of hot and dense nuclear matter
}

\author{
Ji-sheng Chen ${ }^{1,2 *} \quad$ Peng-fei Zhuang ${ }^{1} \quad$ Jia-rong $\mathrm{Li}^{2}$ \\ ${ }^{1}$ Physics Department, Tsinghua University, Beijing 100084,People's Republic of China \\ ${ }^{2}$ Institute of Particle Physics, Hua-Zhong Normal University, Wuhan 430079, People's Republic of China
}

\begin{abstract}
The influence of the in-medium mesons on the effective nucleon mass and in turn on the equation of state of hot/dense nuclear matter is discussed in the Walecka model. Due to the self-consistent treatment of couplings between nucleons and $\sigma$ and $\omega$ mesons, the temperature and density dependence of the effective hadron masses approaches more towards the Brown-Rho scaling law, and the compression modulus $K$ is reduced from $550 \mathrm{MeV}$ in mean field theory to an accepted value $318.2 \mathrm{MeV}$.
\end{abstract}

PACS numbers:21.65.+f 11.10.Wx 21.30.Fe 25.75.-q

The study of strongly interacting nuclear matter under extreme condition realized in relativistic heavy ion collisions has attracted a lot of attention during recent years(for example, see Ref. [1] and references therein). In high energy nuclear collisions the temperature may approach to several hundred MeV's or the density to several times of the normal nuclear density $\rho_{0}$. It is believed that the property of hadrons in such high temperature or density will be quite different from that in vacuum. For instance, the behaviors of the in-medium hadronic masses may be qualitatively expressed by the so-called Brown-Rho scaling law [2].

The quantum hadrodynamics QHD-I model (Walecka model ) and its extensions have been widely used to discuss the property of the symmetric nuclear matter and finite nuclei [3-7]. For example, the saturation properties at the normal density can be explained successfully by the mean field approximation.

In the Walecka model, the nucleons interact with each other through the exchange of $\sigma$ and $\omega$ mesons. The $\sigma$ exchange gives the attractive force, while the $\omega$ exchange the repulsive force. The mean field theory (MFT) has been widely used to discuss the nuclear matter saturation property at the normal density, while it was found that the obtained compression modulus $K \sim 550 \mathrm{MeV}$ is larger than the acceptable value $300 \pm 50 \mathrm{MeV}[8]$. The reason for so high compression modulus is that the effective nucleon mass drops too fast with the density. By taking into account the vacuum fluctuations, the behavior of the effective nucleon mass with density may be cured to some extent, but the obtained compression modulus $K \sim 450 \mathrm{MeV}$ is still larger than the acceptable value. To solve this problem, the $\sigma-\omega$ model including nonlinear interaction terms $b \sigma^{3}+c \sigma^{4}$ of $\sigma$ and the ZimanyiMoszkowski (ZM) model have been proposed $[6,7]$. With more adjustable parameters in the nonlinear $\sigma-\omega$ version, the effective nucleon mass drops slowly and the compression modulus $K$ may be in the region of "appropriate values." The behaviors of the effective nucleon mass and the compression modulus $K$ may be also cured to some extent with the nonrenormalizable ZM model [7].

On the other hand, the in-medium effects of hadrons under extreme environment have been emphasized in the QHD-like framework. Since Walecka model's Lagrangian is a truncated form of a chirally symmetric Lagrangian with the vector and scalar fields taken as chiral-singlet fields $[9,10]$, it is argued that the predictions about the effective masses of hadrons in hot/dense environments by QHD are intrinsically consistent with the chiral symmetry restoration $[11,12]$. This simple model includes implicitly vacuum effects and subnucleon structures. With QHD-I and by considering the Dirac sea contribution, the obtained effective masses of light vector mesons drop down with increasing density/temperature. However, in discussing the hadronic masses, the relevant equations of QHD-I model had not been solved with a consistent manner, i.e., first solving the equations for the effective nucleon mass $M_{N}^{*}$ and chemical potential $\mu_{N}^{*}$ (for finite temperature occasion) in MFT or RHA (relativistic Hartree approximation) approach and then using the obtained $M_{N}^{*}$ and $\mu_{N}^{*}$ to get the full propagators for mesons $\sigma$ and $\omega$ and even $\rho$ [13-16]. Therefore, the in-medium meson effects were not reflected in the effective nucleon mass as pointed out by Bhattacharyya et al. in Ref. [17]. Our motivation is to study the effect of nonperturbative inmedium modification of $\sigma$ and $\omega$ on the effective nucleon mass and on the equation of state (EOS) of hot/dense nuclear matter by using renormalizable original version QHD-I. With Dyson-Schwinger Green function approach, the in-medium resummed nucleon and meson propagators are treated self-consistently.

The full description for the Lagrangian of Walecka model can be found in Refs. $[4,18]$. Under the mean field approximation, the full nucleon propagator in the medium as indicated in Fig.1 is attributed to the calculation of the tadpole self-energies with finite temperature field theory:

*Email address: chenjs@iopp.ccnu.edu.cn 


$$
\begin{aligned}
& \Sigma_{s}=-\frac{g_{\sigma}^{2}}{m_{\sigma}^{2}} T \sum_{p_{0}} \int_{\mathbf{p}} \operatorname{Tr} \frac{1}{p-M_{N}^{*}}, \\
& \Sigma_{v}=-\frac{g_{\omega}^{2}}{m_{\omega}^{2}} \gamma^{\mu} T \sum_{p_{0}} \int_{\mathbf{p}} \operatorname{Tr} \gamma_{\mu} \frac{1}{\not p-M_{N}^{*}},
\end{aligned}
$$

where $p_{0}=(2 n+1) \pi T i+\mu_{N}^{*}$ with $T$ and $\mu_{N}^{*}$ being temperature and effective baryon chemical potential, respectively, and the symbol $\int_{\mathbf{p}}=\int \frac{d^{3} \mathbf{p}}{(2 \pi)^{3}}$. With the residue theorem, one can divide the self-energies into the vacuum fluctuation and the matter(obviously related to the distribution functions) parts, and define the effective nucleon mass $M_{N}^{*}$ and effective chemical potential $\mu_{N}^{*}[4]$,

$$
\begin{aligned}
M^{*}-M & =-\frac{\gamma g_{\sigma}^{2}}{m_{\sigma}^{2}} \int_{\mathbf{p}} \frac{M_{N}^{*}}{E_{N}^{*}}\left(n_{N}+\bar{n}_{N}\right)+\Delta M_{v a c}^{*} \\
\mu_{N}^{*}-\mu_{N} & =-\frac{g_{\omega}^{2}}{m_{\omega}^{2}} \rho_{B}
\end{aligned}
$$

where the baryon density

$$
\rho_{B}=\gamma \int_{\mathbf{p}}\left(n_{N}-\bar{n}_{N}\right)
$$

with

$$
\begin{aligned}
& n_{N}=\frac{1}{e^{\beta\left(E_{N}^{*}-\mu_{N}^{*}\right)}+1}, \quad \bar{n}_{N}=\frac{1}{e^{\beta\left(E_{N}^{*}+\mu_{N}^{*}\right)}+1}, \\
& E_{N}^{*}=\sqrt{p^{2}+M_{N}^{* 2}} .
\end{aligned}
$$

The spin-isospin degenerate factor is $\gamma=4$ for symmetric nuclear matter and 2 for neutron star.

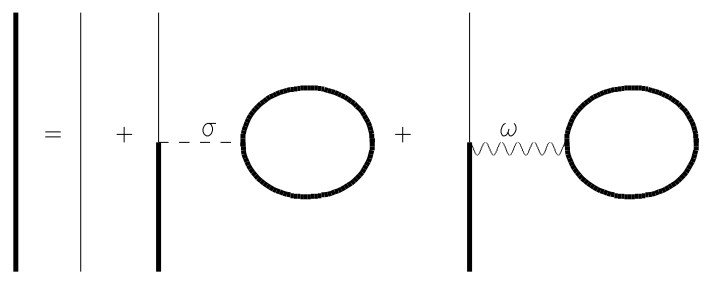

FIG. 1. Diagrammatic representation for the nucleon propagator in relativistic Hartree approximation (RHA).

One can see that the self-consistent equation of effective nucleon mass in RHA is just the result in the MFT plus the vacuum fluctuation contribution $\Delta M_{v a c}^{*}$. Similarly, the energy density and the pressure are the MFT results plus the vacuum fluctuation contribution $\Delta \varepsilon_{v a c}^{*}$,

$$
\begin{aligned}
\varepsilon= & \frac{m_{\sigma}^{2}}{2 g_{\sigma}^{2}}\left(M_{N}-M_{N}^{*}\right)^{2}+\frac{g_{\omega}^{2}}{2 m_{\omega}^{2}} \rho_{B}^{2}+ \\
& \gamma \int_{\mathbf{p}} E_{N}^{*}\left(n_{N}+\bar{n}_{N}\right)-\Delta \varepsilon_{v a c}^{*} ; \\
P=- & \frac{m_{\sigma}^{2}}{2 g_{\sigma}^{2}}\left(M_{N}-M_{N}^{*}\right)^{2}+\frac{g_{\omega}^{2}}{2 m_{\omega}^{2}} \rho_{B}^{2}- \\
& \gamma T \int_{\mathbf{p}}\left[\ln \left(1-n_{N}\right)+\ln \left(1-\bar{n}_{N}\right)\right]+\Delta \varepsilon_{v a c}^{*}+B^{*} .
\end{aligned}
$$

In Eqs. (3), (6) and (7), the explicit expressions for the vacuum fluctuation contributions $\Delta M_{v a c}^{*}$ and $\Delta \varepsilon_{v a c}^{*}$ can be found in Refs. $[4,19]$. The thermodynamics compensatory term $B^{*}$ in Eq. (7) can be determined uniquely by the thermodynamics self-consistency relation between the energy density $\varepsilon$ and pressure $p$. For MFT and RHA approaches, this term is vanishing.

The properties of mesons are studied by the meson propagators in medium, which are normally calculated by using the random phase approximation (RPA) with the full nucleon propagator in Fig.1. The Dyson-Schwinger equation of the $\omega$ meson propagator is indicated in Fig.2 and its solution for the full propagator $D^{\mu \nu}$ can be determined by the polarization tensor $\Pi^{\mu \nu}(k)$,

$$
\Pi^{\mu \nu}(k)=\left(D^{-1}\right)^{\mu \nu}-\left(D_{(0)}^{-1}\right)^{\mu \nu},
$$

where $D_{(0)}^{\mu \nu}$ is the bare propagator. Using the Feynman rules at finite temperature [20], one has

$$
\Pi^{\mu \nu}(k)=2 g_{\omega}^{2} T \sum_{p_{0}} \int_{\mathbf{p}} \operatorname{Tr}\left[\gamma^{\mu} \frac{1}{p-M_{N}^{*}} \gamma^{\nu} \frac{1}{p-\not k-M_{N}^{*}}\right] .
$$

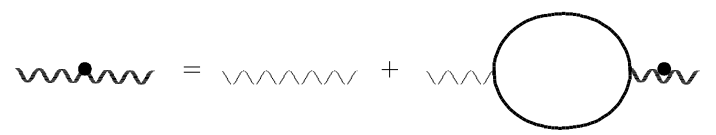

FIG. 2. The in-medium $\omega$ propagator in random phase approximation.

Different from the occasion in vacuum, there are two independent elements $\Pi^{L(T)}$ for the polarization tensor $\Pi^{\mu \nu}(k)$ in medium,

$$
\begin{aligned}
\Pi^{\mu \nu}(k)= & \Pi^{L}(k) P_{L}^{\mu \nu}+\Pi^{T}(k) P_{T}^{\mu \nu}, \\
D^{\mu \nu}= & -\frac{P_{L}^{\mu \nu}}{k^{2}-m_{\omega}^{2}-\Pi_{L}(k)} \\
& -\frac{P_{T}^{\mu \nu}}{k^{2}-m_{\omega}^{2}-\Pi_{T}(k)}-\frac{k^{\mu} k^{\nu}}{m_{\omega}^{2} k^{2}},
\end{aligned}
$$

where the $P_{L}^{\mu \nu}$ and $P_{T}^{\mu \nu}$ are the standard projection tensors [20]. The ingredients $\Pi_{L}(k)$ and $\Pi_{T}(k)$ are determined by

$$
\Pi_{L}(k)=\frac{k^{2}}{\mathbf{k}^{2}} \Pi^{00}(k), \quad \Pi_{T}(k)=\frac{1}{2} P_{T}^{i j} \Pi_{i j}(k) .
$$

The pole position of the full propagator $D^{\mu \nu}$ determines completely the dispersion relation of $\omega$ meson excitations in medium. The expressions for the various components of $\Pi^{\mu \nu}(k)$ are similar to those of $\rho$ as given in Refs. [18,21] except the vanishing of the tensor coupling constant for $\omega$ meson, i.e., $\kappa_{\omega}=0$.

Analogously to the discussion of $\omega$ meson, one can discuss the property of $\sigma$ in the medium. Its diagrammatic 
Dyson-Schwinger equation is similar to Fig. 2 and the self-energy is $[21,22]$

$$
\begin{aligned}
\Pi(k)= & 2 g_{\sigma}^{2} T \sum_{p 0} \int_{\mathbf{p}} \operatorname{Tr}\left[\frac{1}{p-M_{N}^{*}} \frac{1}{p-\not k-M_{N}^{*}}\right] \\
= & \frac{3 g_{\sigma}^{2}}{2 \pi^{2}}\left[3\left(M_{N}^{*^{2}}-M_{N}^{2}\right)-4\left(M_{N}^{*}-M_{N}\right) M_{N}\right. \\
& -\left(M_{N}^{*^{2}}-M_{N}^{2}\right) \int_{0}^{1} \ln \frac{M_{N}^{*^{2}}-x(1-x) k^{2}}{M_{N}^{2}} d x \\
& \left.-\int_{0}^{1}\left(M_{N}^{2}-x(1-x) k^{2}\right) \ln \frac{M_{N}^{*^{2}}-x(1-x) k^{2}}{M_{N}^{2}-x(1-x) k^{2}} d x\right] \\
& +\frac{g_{\sigma}^{2}}{\pi^{2}} \int \frac{p^{2} d p}{E_{N}^{*}}\left(n_{N}+\bar{n}_{N}\right)\left[2+\frac{k^{2}-4 M_{N}^{*}}{4 p|\mathbf{k}|}(a+b)\right],
\end{aligned}
$$

where

$a=\ln \frac{k^{2}-2 p|\mathbf{k}|-2 k_{0} E_{N}^{*}}{k^{2}+2 p|\mathbf{k}|-2 k_{0} E_{N}^{*}}, \quad b=\ln \frac{k^{2}-2 p|\mathbf{k}|+2 k_{0} E_{N}^{*}}{k^{2}+2 p|\mathbf{k}|+2 k_{0} E_{N}^{*}}$.

There are two kinds of effective meson masses related to our work. One is the pole mass $m^{*}$, which can be matched to the results obtained by such as QCD sum rules. It is defined by the pole position of the meson propagator in medium by taking the limit $|\mathbf{k}| \rightarrow 0$ of $\Pi(k)$,

$$
k_{0}^{2}-m^{2}-\lim _{|\mathbf{k}| \rightarrow 0} \Pi(k)=0 .
$$

The other is the off-shell mass $\bar{m}$ in the medium determined by

$$
\bar{m}^{2}=m^{2}+\lim _{k_{0} \rightarrow 0} \lim _{|\mathbf{k}| \rightarrow 0} \Pi(k) .
$$

Due to the fact that the longitudinal and transverse components of the $\omega$ polarization tensor $\Pi_{\omega}^{\mu \nu}(k)$ coincide with each other in taking the limit $|\mathbf{k}| \rightarrow 0$, the subscripts $L$ and $T$ for $\Pi_{\omega}(k)$ are omitted.

As calculated in the following, the meson property will be quite different from the vacuum scenario. To consider the back interactions of $\sigma$ and $\omega$ mesons with nucleon $N$ from the point of view of self-consistency, the in-medium $\sigma$ and $\omega$ propagators with vanishing four-momentum transfer should be used in determining the nucleon propagator as indicated by Fig.1 due to the adapted Hartree approximation. Therefore, the meson masses $m_{\sigma}$ and $m_{\omega}$ in the effective nucleon mass and chemical potential equations (3) and (4), and in the EOS's (6) and (7) should be the off-shell meson masses $\bar{m}$ 's determined by Eqs. (14). It means that Eqs. (3) and (4) obtained by the RHA of the nucleon propagator and Eqs. (14) obtained by the RPA of the meson propagator form a closed set of equations, and should be solved simultaneously.
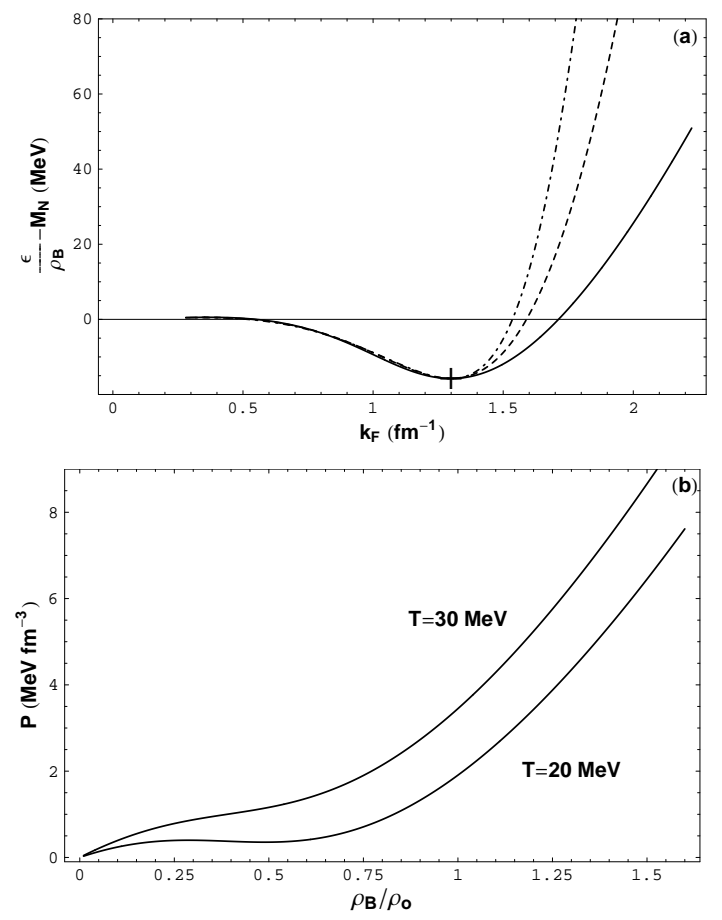

FIG. 3. (a) The binding energy for nuclear matter as a function of Fermi momentum at $T=0$. The dot-dashed, dashed, and solid lines represent the MFT, RHA, and our results, respectively; (b) The pressure as a function of rescaled density $\rho_{B} / \rho_{0}$ for two temperatures $T=20 \mathrm{MeV}$ and $30 \mathrm{MeV}$.

Considering the in-medium effects of the off-shell masses (14) on the EOS, one must at first refit the binding energy at the normal nuclear density at zero temperature by adjusting the coupling constants $g_{\omega(\sigma)}$ as done in MFT and RHA. The coupling constants are listed in Table I, which are smaller than those of MFT and RHA. It is interesting to note that the EOS becomes much softer and the compression modulus

$$
K=\left.9 \rho_{B}^{2} \frac{\partial^{2} e_{N}}{\partial \rho_{B}^{2}}\right|_{\rho_{B}=\rho_{0}}
$$

is reduced to $K=318.2 \mathrm{MeV}$, which is acceptable in dealing with realistic nuclear matter. In Eq. (15), $e_{N}$ is the binding energy $\left(\varepsilon / \rho_{B}-M_{N}\right)$. Of course, this interesting numerical result can be also obtained with the nonlinear $\sigma-\omega$ version by adjusting the additional parameters due to the renormalizability of original QHD-I as pointed out in the introduction [5]. However, it is interesting enough that it has been obtained here from the point of view of in-medium meson contribution on the nucleon and bulk property of nuclear matter. To the best of our knowledge, a similar numerical result of compression modulus $K$ has also been obtained long ago by Ji in Ref. [23] with QHD-I through taking into account the polarization effects at zero temperature. It should be pointed out that our numerical results (especially $g_{\sigma}, g_{\omega}$, and $K$ ) are also different from those of Ref. [17] for the zero-temperature scenario. 
One can further study the energy density and pressure. One should note that the consideration of the back interactions of in-medium mesons with nucleons will lead to the nonvanishing compensatory term $B^{*}$ in Eq. (7) due to the implicit higher order contribution from the resummed meson propagators instead of frozen ones. In principle, this term can be expanded into a series of $M_{N}^{*}-M_{N}$. With the energy density Eq. (6) and thermodynamics self-consistency condition at zero temperature $p=\rho_{B}^{2}\left(\partial / \partial \rho_{B}\right)\left(\varepsilon / \rho_{B}\right)$, this compensatory term can be fixed uniquely. In the upper panel (a) of Fig.3, we give out the binding energy at $T=0$, from which one can confirm that the equation of state really becomes softer and the compression modulus is much smaller than the results of MFT and RHA. The first order liquid-gas phase transition in the low temperature case still exists as indicated by Fig.3(b) and the critical temperature $T_{c}$ is about $21 \mathrm{MeV}$.
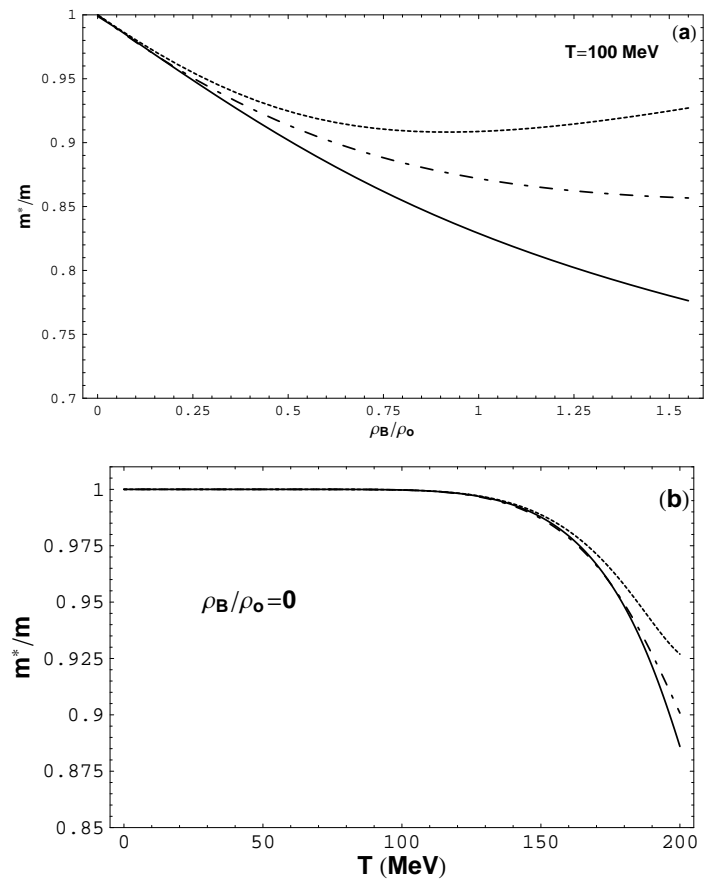

FIG. 4. The effective masses $M_{N}^{*}$ (solid lines ), $m_{\sigma}^{*}$ (dotted lines) and $m_{\omega}^{*}$ (dot-dashed lines) as functions of rescaled density $\rho_{B} / \rho_{0}$ at $T=100 \mathrm{MeV}$ (a) and temperature $T$ at $\rho_{B}=0$ (b), respectively.

The pole masses $M_{N}^{*}, m_{\sigma}^{*}$ and $m_{\omega}^{*}$ determined by Eqs. (3) and (13) are indicated in Fig.4. Because of considering the back interactions of $\sigma$ and $\omega$ mesons with nucleons, their temperature and density dependence approaches more towards the Brown-Rho scaling law compared with the results of MFT/RHA. The key point is that the exchanged mesons are not bare but in-medium ones, their masses are self-consistently determined by the Dyson-Schwinger equations. The temperature and density dependence of hadron masses studied here is analogous to that of hidden local symmetry theory of Harada and Yamawaki where such dependence is required by the Wilsonian matching to QCD [24]. It is interesting to note that our results are consistent with those obtained in recent works $[25,26]$, where the Brown-Rho scaling fits naturally into the relevant framework. This consistency is attributed to the hidden chiral symmetry in QHD-I and should contribute to the understanding of hadronic matter under extreme conditions within the relativistic nuclear theory framework.

In summary, we have investigated the in-medium meson contribution to the EOS of hot/dense nuclear matter with the original Walecka model by treating the coupled nucleon and meson propagators self-consistently. This kind of contribution makes the EOS softer than the RHA and MFT approaches. Due to the couplings between nucleon and in-medium mesons, the compression modulus $K$ drops from about $550 \mathrm{MeV}$ to about $320 \mathrm{MeV}$ and the effective hadronic masses approach more towards the Brown-Rho scaling law, and the low temperature liquidgas phase transition still exists.

Acknowledgements: Beneficial communications and discussions with Professor S. Phatak, Professor S. K. Ghosh, and Professor Zh.-X Li are acknowledged by one of the authors (J.-S Chen). This work was supported by the NSFC under Grants Nos. 10135030, 10175026, 19925519 and the China Postdoc Research Fund.

TABLE I. The coupling constants $g_{\sigma}$ and $g_{\omega}$, the effective nucleon mass $M_{N}^{*}$ and the compression modulus $K$ at normal nuclear density $\rho_{0}=0.1484 \mathrm{fm}^{-3}$ at $T=0$. In MFT, RHA, and our approach(labeled as RHA+RPA), the nucleon and $\omega$ meson masses in vacuum are taken to be $M_{N}=939 \mathrm{MeV}, m_{\omega}=783 \mathrm{MeV}$, and the $\sigma$ meson mass in the vacuum is selected to be $m_{\sigma}=520 \mathrm{MeV}$ (in the literature with MFT) and $458 \mathrm{MeV}$. To compare with the results in MFT and RHA, we have also shown the medium dependent coupling constants $C_{s}^{2}=g_{\sigma}^{2}\left(M_{N}^{2} / \bar{m}_{\sigma}^{2}\right)$ and $C_{v}^{2}=g_{\omega}^{2}\left(M_{N}^{2} / \bar{m}_{\omega}^{2}\right)$.

\begin{tabular}{cccccccc}
\hline \hline & $g_{\sigma}^{2}$ & $g_{\omega}^{2}$ & $m_{\sigma}(\mathrm{MeV})$ & $C_{s}^{2}$ & $C_{v}^{2}$ & $K \quad(\mathrm{MeV})$ & $\frac{M_{N}^{*}}{M_{N}}$ \\
\hline MFT & 109.94 & 191.04 & 520 & 358.49 & 274.76 & 547.2 & 0.540 \\
& 85.286 & 191.04 & 458 & 358.49 & 274.76 & 547.2 \\
\hline RHA & 54.289 & 102.76 & 458 & 228.19 & 147.78 & 0.540 \\
\hline RHA+RPA & 42.229 & 69.729 & 458 & 152.59 & 86.263 & 318.2 & 0.731 \\
\hline \hline
\end{tabular}


[1] R. Rapp and J. Wambach, Adv. Nucl. Phys. 25, 1 (2000).

[2] G. E. Brown and M. Rho, Phys. Rev. Lett. 66, 2720 (1991).

[3] J.D. Walecka, Ann. Phys. (N.Y.) 83, 491 (1974).

[4] B.D. Serot and J.D. Walecka, Adv. Nucl. Phys. 16, 1 (1986).

[5] B.D. Serot and J.D. Walecka, Int. J. Mod. Phys. E 6, 515 (1997).

[6] J. Boguta and A.R. Bodmer, Nucl. Phys. A 292, 413 (1977).

[7] J. Zimanyi and S.A. Moszkowski, Phys. Rev. C 42, 1416 (1990).

[8] H. Kouno, K. Koide, T. Mitsumori, N. Noda, A. Hasegawa, and M. Nakano, Phys. Rev. C 52, 135 (1995).

[9] G. Gelmini and B. Ritzi, Phys. Lett. B 357, 431 (1995).

[10] G.E. Brown and M. Rho, Nucl. Phys. A 596, 503 (1996).

[11] R. Aguirre, Phys. Rev. C 63, 025206 (2001).

[12] T.D. Cohen, R.J. Furnstahl, and D.K. Griegel, Phys. Rev. Lett. 67, 961 (1991).

[13] H. Shiomi and T. Hatsuda, Phys. Lett. B 334, 281 (1994); T. Hatsuda, H. Shiomi, and H. Kuwabara, Prog.
Theor. Phys. 95, 1009 (1996).

[14] H.-C. Jean, J. Piekarewicz, and A. G. Williams, Phys. Rev. C 49, 1981 (1994).

[15] A. K. Dutt-Mazumder, B. Dutta-Roy, and A. Kundu, Phys. Lett. B 399, 196 (1997); S. Sarkar, J. Alam, P. Roy, A.K. Dutt-Mazumder, B. Dutt-Roy, and B. Sinha, Nucl. Phys. A 634, 206 (1998).

[16] Y. Iwasaki, H. Kouno, A. Hasegawa, and M. Nakano, Int. J. Mod. Phys. E 9, 459 (2000).

[17] A. Bhattacharyya, S.K. Ghosh, and S.C. Phatak, Phys. Rev. C 60, 044903 (1999).

[18] Ji-sheng Chen, Jia-rong Li, and Peng-fei Zhuang, J. High Energy Phys. 11, 014 (2002).

[19] S.A. Chin, Ann. Phys. (N.Y.) 108, 301 (1977).

[20] J.I. Kapusta, Finite Temperature Field Theory (Cambridge University Press, Cambridge, England, 1989).

[21] Ji-sheng Chen, Jia-rong Li, and Peng-fei Zhuang, Phys. Rev. C 67, 068202 (2003).

[22] J.C. Caillon and J. Labarsouque, Phys. Lett. B 311, 19 (1993).

[23] Xiang-Dong Ji, Phys. Lett. B 208, 19 (1988).

[24] M. Harada and K. Yamawaki, Phys. Rev. Lett. 83, 3374 (1999); Phys. Rev. D 64, 014023 (2001) and references therein.

[25] G.E. Brown and M. Rho, Phys. Rep. 363, 85 (2002).

[26] C. Song, Phys. Rep. 347, 289 (2001). 